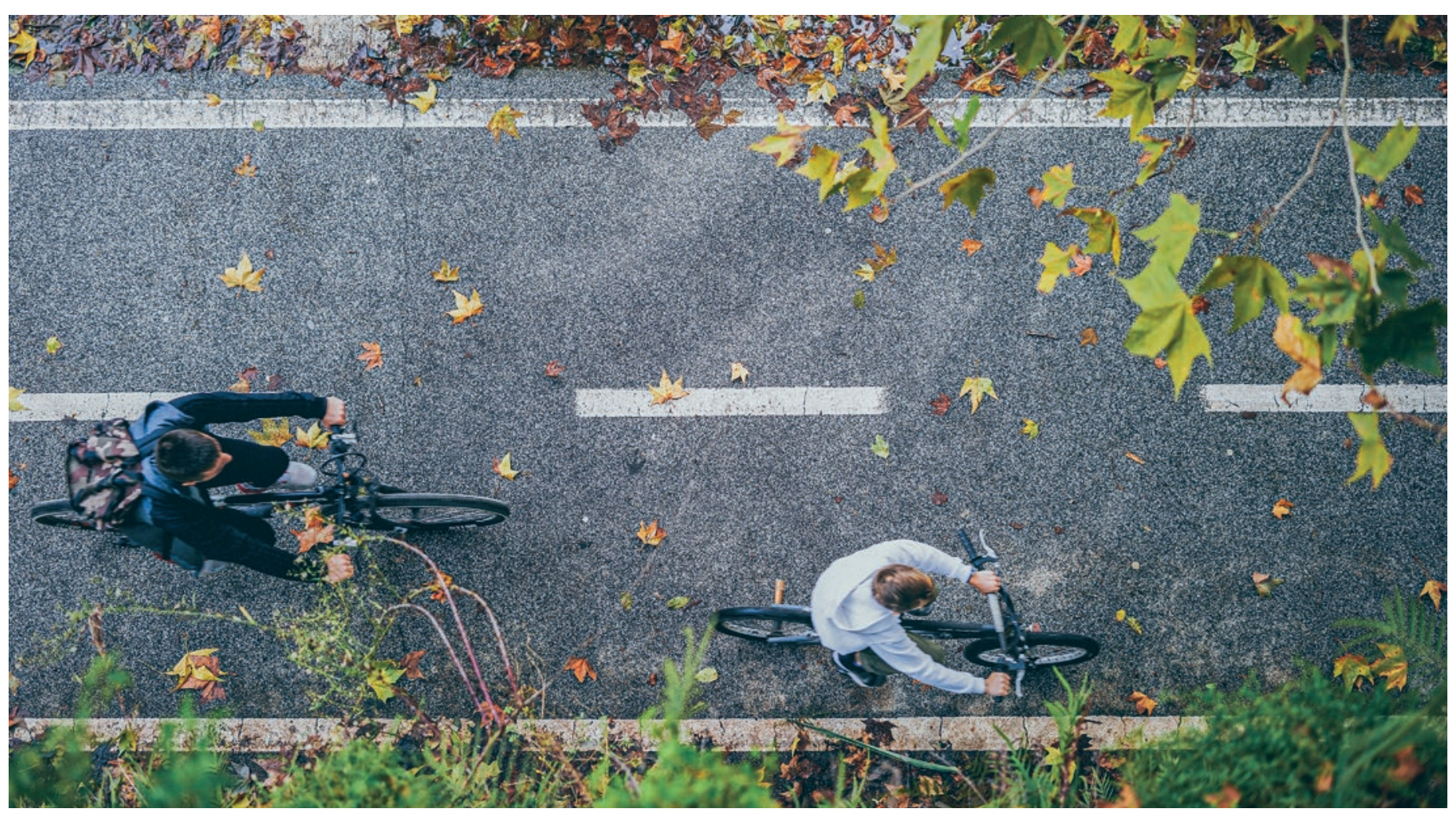

\title{
Le chemin se fait en marchant
}

\section{Robin Riesera, Carlos Quintob ${ }^{\text {, Barbara Weilc }}$}

${ }^{a}$ Collaborateur scientifique, division Santé publique de la FMH; ${ }^{b}$ Membre du Comité central de la FMH, responsable du département Santé publique et professions de la santé; ${ }^{c}$ Cheffe de la division Santé publique et professions de la santé

Le changement climatique est omniprésent. Le secteur de la santé contribue largement aux émissions de gaz à effet de serre qui l'alimentent. Afin d'assumer la responsabilité commune en matière de changement climatique, la FMH a élaboré la stratégie "Santé planétaire: stratégie du corps médical suisse sur les possibilités d'action concernant le changement climatique». Celle-ci a été adoptée par la Chambre médicale le 7 octobre 2021.

L'humanité a suivi ces dernières décennies un chemin qui semble confirmer l'aphorisme attribué à Franz Kafka «Le chemin se fait en marchant» car, aujourd'hui, le changement climatique est bien là - en Suisse comme dans l'esprit de la population. Les catastrophes naturelles de l'été 2021 ont provoqué des dégâts majeurs et des pertes humaines dans une grande partie de l'Europe. Ces situations climatiques extrêmes de plus en plus fréquentes sont à attribuer au changement du climat et donc également au changement des écosystèmes en Suisse. Tous deux ont des effets directs et indirects sur la santé de la population de notre pays (fig. 1). Les températures élevées impactent l'organisme humain. La chaleur peut être à l'origine de sensations d'épuisement et de coups de chaleur; elle peut aussi aggraver des maladies déjà présentes, telles que les affec- tions cardiovasculaires, respiratoires et rénales ainsi que les troubles mentaux [1]. De plus, les périodes de chaleur augmentent le risque d'infections et de troubles de la cicatrisation [2]. Les vagues de chaleur et les journées tropicales engendrent en Suisse non seulement une augmentation des admissions aux urgences hospitalières [3], mais aussi une hausse de la mortalité générale [4, 5]. Les nuits tropicales constituent un risque supplémentaire pour la santé, le repos nocturne étant troublé par le manque de fraîcheur [6].

\section{La chaleur fait augmenter les agressions}

Selon différentes études, les températures élevées ont également un impact négatif sur la santé mentale. Des études menées aux Etats-Unis et en Australie ont mon- 


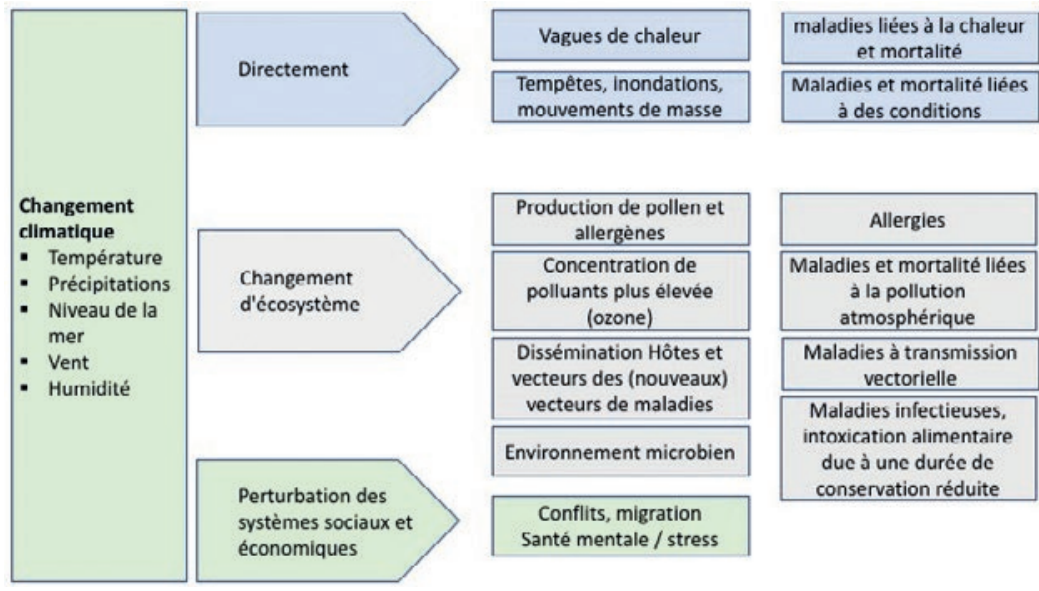

Figure 1: Effets directs et indirects du changement climatique sur la santé.

Source: Swiss TPH, adapté de [8-10].

tré que les agressions et le taux de criminalité augmentaient pendant les vagues de chaleur. Conséquence de la dégradation des écosystèmes, la propagation possible de vecteurs, agents pathogènes et plantes allergènes augmente les risques pour la santé. En outre, les maladies infectieuses transmises par les aliments, l'eau et les vecteurs pourraient devenir plus fréquentes. Dans une large mesure, la production et la conservation des aliments dépendent des conditions climatiques, si bien que l'hygiène alimentaire revêt une importance croissante.

Le secteur de la santé contribue sensiblement aux émissions de gaz à effet de serre des différents pays. Sur le plan mondial, la part du secteur de la santé par rapport aux émissions totales s'élève à $4,6 \%$ d'après le dernier Lancet Countdown [1]. La part des émissions de $\mathrm{CO}_{2}$ du système de santé est particulièrement élevée p. ex.aux Etats-Unis avec 8 à 10\% [6, 7]. En Grande-Bretagne, elle était de 4 à 5\% en 2017 mais baisse depuis cette date compte tenu des efforts pour que le National

\section{Le changement climatique est bien là - en Suisse et dans l'esprit de la population.}

Health Service (NHS) [8] devienne le premier système de santé avec zéro émission [1]. Les chiffres pour la Suisse varient selon l'étude et la méthode de calcul employée.

Une comparaison internationale de l'empreinte $\mathrm{CO}_{2} \mathrm{du}$ secteur de la santé de Pichler et al. [9] estime que la part pour la Suisse était de 5,9\% en 2014. Health Care without Harm a estimé cette valeur à 6,7\% pour 2014 [10]. Jusqu'ici, on ne dispose d'aucun chiffre d'études nationales. Depuis la publication du sixième rapport d'évaluation du Groupe d'experts intergouvernemental sur l'évolution du climat (GIEC), on ne peut plus nier l'évidence du changement climatique et de ses conséquences [11]. La responsabilité incombe désormais aux organisations, aux institutions et aux entreprises mais aussi aux citoyennes et aux citoyens. Atténuer le changement climatique - et donc protéger notre santé et celle des prochaines générations - devrait déjà guider nos actions aujourd'hui.

\section{Chacun a sa part de responsabilité}

Les progrès réalisés un peu partout dans le monde en matière de santé risquent d'être compromis par le réchauffement climatique. Les inondations, les fortes pluies, les incendies de forêts, la sécheresse de l'été 2021 et le sixième rapport d'évaluation du GIEC [11] publié en août 2021 ont clairement montré que chacune et chacun doit assumer sa part de responsabilité dans la protection du climat et de la santé publique.

Il y a déjà 10 ans, le NHS s'est engagé sur la voie de la protection du climat [8]. Ses efforts se concentrent sur les organisations et les individus, par exemple sur les médecins de famille. Le Green Impact for Health Toolkit du Royal College of General Practicioners (RCGP) propose

\section{Selon différentes études, les températures} élevées ont également un impact négatif sur la santé mentale.

des idées sur différents thèmes afin d'intégrer la durabilité dans le travail quotidien d'un cabinet. Il décrit des mesures axées sur les objectifs de développement durable (ODD) de l'ONU, qui peuvent être mises en œuvre étape par étape en fonction des besoins et des possibilités. Par exemple dans le domaine des déchets et du recyclage, si un cabinet trie ses déchets, utilise du papier recyclé et recycle ses déchets de papier, il atteint le niveau bronze en matière de déchets et de recyclage. Les autres niveaux (argent, or et carbone) comprennent la réduction du plastique à usage unique et du matériel de protection individuelle, la gestion des médicaments non utilisés, la gestion des stocks et des commandes, le choix des fournitures de bureau, etc.

Si les mesures sont mises en œuvre dans l'ensemble des 13 domaines, il est même possible, dans le cadre d'une procédure d'audit, de faire certifier son propre cabinet ou de recevoir le Green Impact award. La possibilité de mettre en œuvre les mesures de manière progressive et la facilité d'accès via une plateforme en ligne font du Green Impact for Health Toolkit du RCGP une mesure proportionnée et réaliste pour promouvoir la réalisation des objectifs de développement durable et la protection du climat. 


\section{Les mesures en Suisse}

Aujourd'hui déjà, des projets innovants portant sur la mise en œuvre de ces objectifs sont en cours dans toute la Suisse. L'Hôpital cantonal de Lucerne a lancé entre 2017 et 2020 un projet de réduction du gaspillage alimentaire. La mise en place de quelques mesures a permis d'atteindre une réduction de $35 \%$, soit environ 150000 tonnes de déchets alimentaires en moins. Le «Site 2000 watts» a pour objectif de créer des zones résidentielles offrant durabilité et efficacité énergétique et inclut dans la planification des aspects tels que la mobilité, les espaces verts, l'utilisation, les matériaux de construction et l'énergie.

Le projet Green Hospital évalue l'efficacité avec laquelle les hôpitaux suisses utilisent leurs ressources afin de déterminer quels processus peuvent être optimisés et quel potentiel d'économie écologique et économique

\section{Les progrès réalisés un peu partout dans le monde en matière de santé risquent d'être compromis par le réchauffement climatique.}

existe. Le 26 septembre 2021, le canton de Berne est devenu le deuxième canton à décider d'inscrire la protection du climat dans sa constitution. Il entend ainsi atteindre la neutralité climatique d'ici à 2050 et collaborer activement avec les communes pour limiter le changement climatique et ses effets néfastes. Ces projets s'inscrivent dans une démarche d'utilisation durable de nos ressources et montrent qu'il est possible d'être respectueux du climat tout en restant compétitif.

\section{"Manifeste suisse pour un avenir sain»}

L’Assemblée des délégués de la FMH a reconnu le changement climatique comme une menace substantielle pour la santé régionale et mondiale lors de sa séance du 3 septembre 2020 et a signé le "Manifeste suisse pour un avenir sain" au nom du corps médical. Afin d'assumer la responsabilité commune en matière de changement climatique, la FMH a élaboré la stratégie «Santé planétaire: stratégie du corps médical suisse sur les possibilités d'action concernant le changement climatique», adoptée par la Chambre médicale de la FMH le 7 octobre 2021. La stratégie comporte des objectifs dans quatre domaines d'action différents: information, réduction, adaptation et rôle de modèle, et ambitionne de rendre le système de santé suisse durablement favorable à la promotion de la santé tout en étant résilient au changement climatique. Le corps médical suisse soutient le renforcement des mesures contre le changement climatique et contribue à atteindre l'objectif d'un système de santé respectueux de l'environnement par la mise en place de mesures proportionnées et financièrement raisonnables.

Toutefois, afin d'atteindre les objectifs climatiques du Conseil fédéral [12] et de mettre en œuvre les objectifs de développement durable de l'ONU, les efforts individuels des médecins, des hôpitaux ou des cantons et des communes ne suffiront pas. Toutes les parties prenantes doivent travailler ensemble pour faire face à la crise climatique. Ce n'est qu'avec des mesures bien coordonnées que nous pourrons contribuer ensemble à la protection du climat et donc de la santé de toutes les personnes résidant en Suisse et ouvrir la voie à un avenir durable et favorable à la santé.

\section{Références}

1 Watts N, Amann M, Arnell N, et al. The 2020 report of The Lancet Countdown on health and climate change: responding to converging crises. The Lancet. Published online 2020.

2 Aghdassi SJS, Schwab F, Hoffmann P, Gastmeier P. The Association of Climatic Factors with Rates of Surgical Site Infections. Dtsch Aerzteblatt Int. 2019;116.

3 Ragettli MS, Vicedo-Cabrera AM, Flückiger B, Röösli M. Impact of the warm summer 2015 on emergency hospital admissions in Switzerland. Environ Health. 2019;18(66):1-10.

4 Ragettli MS, Röösli M. Effets de la chaleur sur la santé en Suisse et importance des mesures de prévention. Décès dus à la chaleur pendant l'été caniculaire 2019 et comparaison avec les étés 2003 2015 et 2018. Institut Tropical et de Santé Publique Suisse (Swiss TPH); 2020. https://www.nccs.admin.ch/dam/nccs/fr/dokumente/ website/sektoren/gesundheit/Rapport\%20Effets\%20de\%20la\%20 chaleur\%20sur\%20la\%20sant\%C3\%A9\%20en\%20Suisse\%20et\%20 importance\%20des\%20mesures\%20de\%20pr\%C3\%A9vention\%20 (Ragettli\%20 \%20R\%C3\%B6\%C3\%B6sli\%202020).pdf.download. pdf/Rapport\%2OEffets\%2Ode\%2Ola\%2Ochaleur\%20sur\%20la\%20 sant\%C3\%A9\%20en\%20Suisse\%20et\%20importance\%20des\%20 mesures\%20de\%20pr\%C3\%A9vention\%20(Ragettli\%20 \%20 R\%C3\%B6\%C3\%B6sli\%202020).pdf

5 Vicedo-Cabrera AM, Ragettli MS, Schindler C, Röösli M. Excess mortality during the warm summer of 2015 in Switzerland. Swiss Med Wkly. 2016;146:w14379

6 Ragettli MS, Vicedo-Cabrera AM, Schindler C, Röösli M. Exploring the association between heat and mortality in Switzerland between 1995 and 2013. Environ Res. 2017;158C:703-9.

7 OFEV. Risques et opportunités liés au climat. Une synthèse à 'échelle de la Suisse. OFEV. 2017:148.

8 NHS England NI. Delivering a 'Net Zero' National Health Service. Published online 2020. Accessed July 14, 2021. https://www.england.nhs.uk/greenernhs/publication/delivering-a-net-zeronational-health-service/

9 Pichler PP et al. International comparison of health care carbon footprints. Environ Res Lett. 2019;14(6): 064004.

10 Health Care Without Harm US \& Canada. Health care's climate foot print. How the health sector contributes to the global climate crisis and opportunities for action. Published 2019. Accessed January 31, 2021. https://noharm-uscanada.org/ClimateFootprintReport

11 IPCC. Climate Change 2021: The Physical Science Basis. Contribution of Working Group I to the Sixth Assessment Report of the Intergovernmental Panel on Climate Change [Masson-Delmotte V, Zhai P, Pirani A, Connors SL, Péan C, Berger S, Caud N, Chen Y, Goldfarb L, Gomis MI, Huang M, Leitzell K, Lonnoy E, Matthews JBR, Maycock TK, Waterfield T, Yelekçi O, Yu R and Zhou B (Eds.)]. Cambridge University Press. In Press; 2021.

12 OFEV (éd.) 2018: La politique climatique suisse. Mise en oeuvre de l'Accord de Paris. Office fédéral de l'environnement, Berne. Info Environnement $n^{\circ} 1803: 28 \mathrm{p}$ 